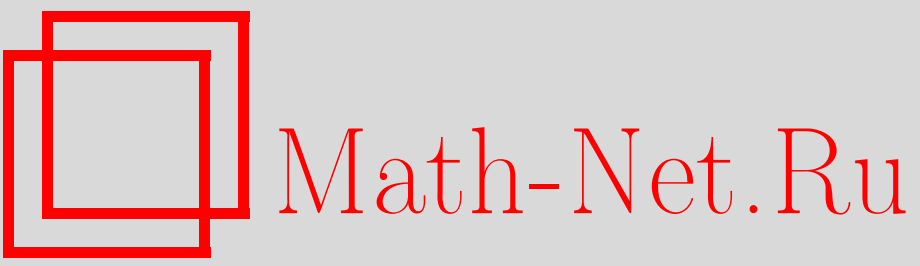

С. А. Зайцев, Структура $S$-матрицы и метод обратной задачи в $J$-матричном подходе, ТМФ, 2004, том 140, номер 1, 29-43

DOI: https://doi.org/10.4213/tmf85

Использование Общероссийского математического портала Math-Net.Ru подразумевает, что вы прочитали и согласны с пользовательским соглашением

http://www.mathnet.ru/rus/agreement

Параметры загрузки:

IP : 35.173 .219 .149

26 апреля 2023 г., 13:38:56 
ТЕОРЕТИЧЕСКАЯ

И МАТЕМАТИЧЕСКАЯ

ФИЗИКА

Том 140, № 1

июль, 2004

(C) 2004 г.

С. А. Зайцев*

\section{СТРУКТУРА $S$-МАТРИЦЫ И МЕТОД ОБРАТНОЙ ЗАДАЧИ В $J$-МАТРИЧНОМ ПОДХОДЕ}

На основе аналитических свойств $S$-матрицы получена система уравнений обратной задачи рассеяния для нелокальных потенциалов с лагерровскими формфакторами. При этом в системе может присутствовать кулоновское отталкивание.

Ключевые слова: сепарабельные потенциалы, обратная задача рассеяния, метод $J$-матрицы.

\section{1. ВВЕДЕНИЕ}

В работах [1] был предложен метод построения по известной $S$-матрице нелокальных взаимодействий

$$
\widehat{V}=\sum_{\alpha, \beta}|\alpha\rangle V^{\alpha \beta}\langle\beta|
$$

с сепарабельными парциальными потенциалами $V^{\alpha \beta}$, задаваемыми разложением

$$
V^{\alpha \beta}=\frac{\hbar^{2}}{2 \mu} \sum_{n=0}^{N_{\alpha}-1} \sum_{n^{\prime}=0}^{N_{\beta}-1}\left|\bar{\phi}_{n}^{(\alpha)}\right\rangle V_{n, n^{\prime}}^{\alpha \beta}\left\langle\bar{\phi}_{n^{\prime}}^{(\beta)}\right|
$$

с использованием осцилляторных [1] базисных функций $\left|\bar{\phi}_{n}^{(\alpha)}\right\rangle$. Здесь $\mu$-приведенная масса, а индекс канала $\alpha$ обозначает набор квантовых чисел, включаюший значение орбитального момента $\ell_{\alpha}$. В работе [2] показано, что подход [1] остается справедливым и в случае, когда взаимодействие включает кулоновское отталкивание, если в качестве формфакторов $\left|\bar{\phi}_{n}^{(\alpha)}\right\rangle$ короткодействуюшего потенциала (2) выбрать лагерровские базисные функции

$$
\left|\bar{\phi}_{n}^{(\alpha)}\right\rangle \equiv\left|\bar{\phi}_{n}^{\ell_{\alpha}}\right\rangle=\frac{n !}{r\left(n+2 \ell_{\alpha}+1\right) !}(2 b r)^{\ell_{\alpha}+1} e^{-b r} L_{n}^{2 \ell_{\alpha}+1}(2 b r),
$$

где $b$ - масштабный параметр.

* Хабаровский государственный технический университет, Хабаровск, Россия. E-mail: zaytsev@mail.khb.ru 
Результат решения обратной задачи в $J$-матричном подходе [1], [2] для произволь-

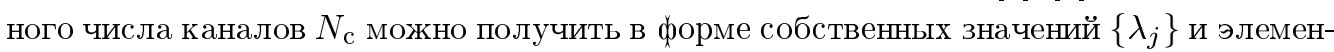

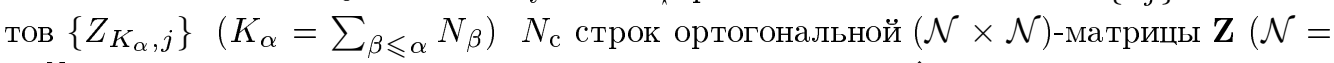
$\left.\sum_{\alpha=1}^{N_{c}} N_{\alpha}\right)$ собственных векторов матрицы гамильтониана $\hat{h}$, рассчитанной в осцилляторном базисе $\left\{\left|\bar{\phi}_{n}^{(\alpha)}\right\rangle, n=\overline{0, N_{\alpha}-1}, \alpha=\overline{1, N_{\mathrm{c}}}\right\}$ [1], или, в случае использования лагерровских формфакторов (3), в базисе ортонормированных функций [2]

$$
\left|\psi_{n}^{(\alpha)}\right\rangle=d_{n}^{\ell_{\alpha}}[2 b r]^{\ell_{\alpha}+1} e^{-b r} L_{n}^{2 \ell_{\alpha}+2}(2 b r), \quad d_{n}^{\ell}=\sqrt{\frac{2 b n !}{(n+2 \ell+2) !}} .
$$

Параметры $\left\{\lambda_{j}, Z_{K_{\alpha}, j}, j=\overline{1, \mathcal{N}}\right\}$ задают набор фазовоэквивалентных потенциалов (1), (2).

С помошью потенциалов (1), (2) конечного ранга $\mathcal{N}$ можно описать данные по рассеянию лишь на ограниченном энергетическом интервале $\epsilon \in\left[0, \epsilon_{0}\right], \epsilon=k^{2}$. Выбор значения правой границы $\epsilon_{0}$ налагает ограничения на параметры задачи $b$ и $N_{\alpha}, \alpha=\overline{1, N_{\mathrm{c}}}$. Например, в одноканальном случае матрица искомого потенциала

$$
\widehat{V}^{\ell}=\frac{\hbar^{2}}{2 \mu} \sum_{n, n^{\prime}=0}^{N-1}\left|\bar{\phi}_{n}^{\ell}\right\rangle V_{n, n^{\prime}}\left\langle\bar{\phi}_{n^{\prime}}^{\ell}\right|
$$

должна обеспечивать сушествование у соответствующей $(N \times N)$-матрицы гамильтониана собственных значений $\lambda_{m}$, удовлетворяющих неравенству $\lambda_{m}>\epsilon_{0}$. Действительно, фаза рассеяния на потенциале (5) (которую назовем теоретической) при $\epsilon>\lambda_{\max }$, где $\lambda_{\max }$ - старшее собственное значение, (неконтролируемо) убывает [2]. Иными словами, в этой области энергий значения теоретической и экспериментальной фаз рассеяния в общем случае различны. В реальных расчетах это различие начинается при меньших энергиях. Таким образом, часть искомых параметров $\left\{\lambda_{j}\right\}$-назовем их "внутренними" - будет принадлежать интервалу $\left[0, \epsilon_{0}\right]$ (где теоретическая и экспериментальная фазы рассеяния совпадают). Остальные - "внешние" - окажутся за пределами интервала $\left[0, \epsilon_{0}\right]$, т.е. в области, где теоретическая и экспериментальная фазы рассеяния заведомо различны. "Внутренние" и "внешние" параметры $\left\{\lambda_{j}, Z_{N, j}\right\}$ различаются способами их поиска. "Внутренние" параметры $\left\{\lambda_{j} \in\left[0, \epsilon_{0}\right], Z_{N, j}^{2}\right\}$ отождествляются с полюсами и вычетами вспомогательной функции $\widetilde{P}$ (матрицы в многоканальном случае) [1], [2], которая однозначно связана с $S$-матрицей. Для определения “внешних" параметров $\left\{\lambda_{j} \notin\left[0, \epsilon_{0}\right], Z_{N, j}^{2}\right\}$ мы не можем воспользоваться данными рассеяния по указанной вьше причине. Здесь мы имеем единственное уравнение - условие нормировки вектора $\left\{Z_{N, j}\right\}$

$$
\sum_{j=1}^{N} Z_{N, j}^{2}=1
$$

В результате в задаче возникают свободные параметры. Уравнения на связанные состояния (в случае присутствия последних) с энергиями $\left\{-\kappa_{\nu}^{2}, \nu=\overline{1, N_{\mathrm{b}}}\right\}$,

$$
\mathcal{D}^{(+)}\left(i \kappa_{\nu}\right)=0
$$


где $\mathcal{D}^{(+)}$- детерминант Фредгольма, не устраняют этой неопределенности, поскольку отвечают за ту часть “внешних" параметров $\left\{\lambda_{j}\right\}$, которые располагаются слева от интервала $\left[0, \epsilon_{0}\right]$.

С ростом числа каналов количество "внешних" параметров возрастает быстрее числа уравнений, которые ограничиваются условиями ортонормированности строк матрицы $\mathbf{Z}$

$$
\sum_{j=1}^{\mathcal{N}} Z_{K_{\alpha}, j} Z_{K_{\beta}, j}=\delta_{\alpha \beta}
$$

Ниже обсуждается $S$-матрица в случае, когда короткодействующий потенциал в системе одноименно заряженных частиц задается сепарабельным разложением (1), (2) с лагерровскими формфакторами (3). Показано, что свойства $S$-матрицы позволяют сформулировать дополнительные уравнения для определения искомых параметров $\left\{\lambda_{j}\right.$, $\left.Z_{K_{\alpha}, j}\right\}$, задающих потенциал $(1),(2)$.

В разделах 2-4 рассмотрено потенциальное рассеяние одноименно заряженных частиц. Обобшение на случай многоканального рассеяния, когда энергия одинакова во всех каналах, сделано в разделе 5 . В разделе 6 приводится пример, иллюстрируюший предлагаемый способ учета особенностей $S$-матрицы при построении потенциала (5). Раздел 7 содержит заключение.

\section{2. СТРУКТУРА $S$-МАТРИЦЫ}

В общем случае рассеяния частиц с отличными от нуля зарядами $\mathcal{Z}_{1}$ и $\mathcal{Z}_{2}$ используемые в методе $J$-матришы [3] аналоги кулоновских решений $S_{n}^{\ell}, C_{n}^{\ell(+)}$ задаются выражениями

$$
\begin{aligned}
S_{n}^{\ell}(k) & =\sin \zeta \omega_{\ell}(k) p_{n}^{\ell}(k), \\
C_{n}^{\ell(+)}(k) & =\frac{1}{\omega_{\ell}(k)} q_{n}^{\ell(+)}(k),
\end{aligned}
$$

где

$$
\omega_{\ell}(k)=\frac{|\Gamma(\ell+1+i t)|(2 \sin \zeta)^{\ell}}{e^{\pi t / 2} \xi^{i t}}
$$

и

$$
\begin{aligned}
p_{n}^{\ell}(k)= & \frac{1}{(2 \ell+1) !}(-\xi)^{n}{ }_{2} F_{1}\left(-n, \ell+1+i t ; 2 \ell+2 ; 1-\xi^{-2}\right), \\
q_{n}^{\ell(+)}(k)=- & \frac{n ! \Gamma(\ell+1+i t)}{\Gamma(n+\ell+2+i t)}(-\xi)^{n+1} \times \\
& \times{ }_{2} F_{1}\left(-\ell+i t, n+1 ; n+\ell+2+i t ; \xi^{2}\right),
\end{aligned}
$$

$\xi=e^{i \zeta}=(i b-k) /(i b+k), t=\mathcal{Z} / k, \mathcal{Z}=\mathcal{Z}_{1} \mathcal{Z}_{2} e^{2} \mu / \hbar^{2}$. Мы ограничиваемся рассмотрением рассеяния одноименно заряженных частиц, т.е. полагаем $\mathcal{Z}>0$. В случае, когда наряду с кулоновским имеется короткодействующий потенциал (5), выражение для $S$-матрицы имеет вид [4]:

$$
S=S_{\ell}^{(c)} \bar{S}^{(N)}
$$


где $S_{\ell}^{(c)}=\Gamma(\ell+1+i t) / \Gamma(\ell+1-i t)-$ кулоновская $S$-матрица,

$$
\bar{S}^{(N)}=\frac{C_{N-1}^{\ell(-)}(k)-P_{N}\left(k^{2}\right) J_{N-1, N}(k) C_{N}^{\ell(-)}(k)}{C_{N-1}^{\ell(+)}(k)-P_{N}\left(k^{2}\right) J_{N-1, N}(k) C_{N}^{\ell(+)}(k)} .
$$

Здесь $J_{n, n^{\prime}}(k)$ - элементы трехдиагональной $J$-матрицы [3]-[5]:

$$
\begin{aligned}
J_{n, n}(k) & =\frac{(n+2 \ell+1) !}{n !}\left[\frac{1}{b}(n+\ell+1)\left(b^{2}-k^{2}\right)+2 \mathcal{Z}\right], \\
J_{n, n+1}(k) & =J_{n+1, n}(k)=\frac{(n+2 \ell+2) !}{2 b n !}\left(b^{2}+k^{2}\right) .
\end{aligned}
$$

$P$-функция определена следуюшим образом [2]:

$$
P_{N}\left(k^{2}\right)=\left(d_{N-1}^{\ell}\right)^{2} \sum_{j=1}^{N} \frac{Z_{N, j}^{2}}{k^{2}-\lambda_{j}}
$$

С учетом (14), (15) и соотношения [5]

$$
C_{n}^{\ell(-)}(k)=C_{n}^{\ell(+)}(k)-2 i S_{n}^{\ell}(k), \quad k>0,
$$

выражение (13) можно записать в виде

$$
\bar{S}^{(N)}=1-i \frac{p_{N-1}^{\ell}(k) \mathcal{A}_{N}\left(k^{2}\right)-p_{N}^{\ell}(k) \mathcal{B}_{N}\left(k^{2}\right)}{q_{N-1}^{\ell(+)}(k) \mathcal{A}_{N}\left(k^{2}\right)-q_{N}^{\ell(+)}(k) \mathcal{B}_{N}\left(k^{2}\right)} 2 \sin \zeta \omega_{\ell}^{2}(k),
$$

где

$$
\mathcal{A}_{N}\left(k^{2}\right)=\prod_{j=1}^{N}\left(k^{2}-\lambda_{j}\right), \quad \mathcal{B}_{N}\left(k^{2}\right)=\left(b^{2}+k^{2}\right) \sum_{j=1}^{N}\left[Z_{N, j}^{2} \prod_{\substack{i=1 \\ i \neq j}}^{N}\left(k^{2}-\lambda_{i}\right)\right] .
$$

Ниже кратко остановимся на уравнениях относительно неизвестных параметров $\left\{\lambda_{j}, Z_{N, j}\right\}$, следуюших из необходимости описания свойств дискретного спектра системы.

\section{3. СВЯЗАННЫЕ СОСТОЯНИЯ}

Для описания связанных состояний необходимо выполнить аналитическое продолжение выражения (17) для $S$-матрицы в верхнюю полуплоскость $k$. Заметим, что при $\operatorname{Im} k>0$ гипергеометрические ряды в (11) сходятся, поскольку здесь $|\xi|<1$.

В работе [6] для детерминанта Фредгольма

$$
\mathcal{D}^{(+)}(k)=\operatorname{det}\left(\mathcal{I}-\widehat{G}^{(+)} \widehat{V}\right),
$$


где $\mathcal{I}$ - единичная $(N \times N)$-матрица, $\widehat{V}$ - матрица потенциала $(5), \widehat{G}^{(+)}$- матрица кулоновской функции Грина, рассчитанная в базисе (3), было получено выражение

$$
\begin{aligned}
\mathcal{D}^{(+)}(k)= & -\frac{(N+2 \ell+1) !}{(2 \ell+1) !(N-1) !} \frac{1}{\left(b^{2}+k^{2}\right)^{N}} \times \\
& \times\left(q_{N-1}^{\ell(+)}(k) \mathcal{A}_{N}\left(k^{2}\right)-q_{N}^{\ell(+)}(k) \mathcal{B}_{N}\left(k^{2}\right)\right) .
\end{aligned}
$$

Таким образом, условие (7) обрашения в нуль детерминанта Фредгольма при заданных значениях энергии $\left\{-\kappa_{\nu}^{2}, \nu=\overline{1, N_{\mathrm{b}}}\right\}$ связанных состояний дает $N_{\mathrm{b}}$ уравнений относительно параметров $\left\{\lambda_{j}, Z_{N, j}\right\}$ :

$$
q_{N-1}^{\ell(+)}\left(i \kappa_{\nu}\right) \mathcal{A}_{N}\left(-\kappa_{\nu}^{2}\right)-q_{N}^{\ell(+)}\left(i \kappa_{\nu}\right) \mathcal{B}_{N}\left(-\kappa_{\nu}^{2}\right)=0
$$

К этому следует добавить информацию об асимптотических нормировочных констан$\operatorname{tax}\left\{\mathcal{M}^{(\nu)}, \nu=\overline{1, N_{\mathrm{b}}}\right\}$ - коэффициентах пропорциональности между (нормированными на единицу) волновыми функциями связанных состояний $\Phi_{\ell}\left(i \kappa_{\nu}, r\right)$ и решениями Йоста $f_{\ell}(k, r)$ :

$$
\Phi_{\ell}\left(i \kappa_{\nu}, r\right)=\mathcal{M}^{(\nu)} f_{\ell}\left(i \kappa_{\nu}, r\right)
$$

В случае

$$
f_{\ell}(k, r) \stackrel{r \rightarrow \infty}{\sim} e^{i(k r-t \ln 2 k r)}
$$

имеет место соотношение [7]-[9]

$$
\left.i \operatorname{Res}\right|_{k=i \kappa_{\nu}} S(k)=(-1)^{\ell}\left(\mathcal{M}^{(\nu)}\right)^{2} .
$$

Соотношение (24) с учетом (12), (17) преобразуется в уравнение

$$
\begin{aligned}
& \frac{p_{N-1}^{\ell}(i \kappa) \mathcal{A}_{N}\left(-\kappa^{2}\right)-p_{N}^{\ell}(i \kappa) \mathcal{B}_{N}\left(-\kappa^{2}\right)}{d\left\{q_{N-1}^{\ell(+)}(k) \mathcal{A}_{N}\left(k^{2}\right)-q_{N}^{\ell(+)}(k) \mathcal{B}_{N}\left(k^{2}\right)\right\} /\left.d k\right|_{k=i \kappa_{\nu}}}= \\
& \quad=-i e^{-i \pi \eta_{\nu}} \exp \left\{\eta_{\nu} \ln \left(\frac{b-\kappa_{\nu}}{b+\kappa_{\nu}}\right)^{2}\right\}\left(\frac{b^{2}-\kappa_{\nu}^{2}}{4 b \kappa_{\nu}}\right)^{2 \ell+1}\left(\frac{\mathcal{M}^{(\nu)}}{\Gamma\left(\ell+1+\eta_{\nu}\right)}\right)^{2},
\end{aligned}
$$

где $\eta_{\nu}=\mathcal{Z} / \kappa_{\nu}$.

Таким образом, стандартный учет информации о дискретном спектре системы дает $2 N_{\mathrm{b}}$ уравнений $(21)$ и $(25)$, которые можно ассоциировать с "внешними" параметрами $\left\{\lambda_{i}<0, Z_{N, i}, i=\overline{1, N_{\mathrm{b}}}\right\}$. Ниже мы рассмотрим особенности $S$-матрицы (17), которые позволяют сформулировать уравнения относительно "внешних" параметров $\left\{\lambda_{j}, Z_{N, j}\right\}$, для которых $\lambda_{j}>\epsilon_{0}$.

2 Теоретическая и математическая физика, т. 140, № 1, 2004 г. 


\section{4. ДОПОЛНИТЕЛЬНЫЕ УРАВНЕНИЯ}

Рассмотрим интегралы

$$
\int_{0}^{\pi} d \zeta\left(C_{m}^{\ell(-)}-\bar{S}^{(N)} C_{m}^{\ell(+)}\right)\left(C_{n}^{\ell(-)}-\bar{S}^{(N)} C_{n}^{\ell(+)}\right)^{*},
$$

характерные для дискретных форм обратной задачи (см., например, книгу [10] и цитируемую в ней литературу). Заметим, что участку $[0, \pi]$ интегрирования по $\zeta$ соответствует бесконечный интервал $k \in[0, \infty)$ (поскольку $\sin \zeta=2 b k /\left(b^{2}+k^{2}\right), \cos \zeta=$ $\left(b^{2}-k^{2}\right) /\left(b^{2}+k^{2}\right)$, см. (11)), и можно воспользоваться соотношением

$$
C_{n}^{\ell(-)}(k)=\left(C_{n}^{\ell(+)}(k)\right)^{*}, \quad k>0
$$

и унитарностью $S$-матрицы: $\bar{S}^{(N)^{*}}=\left(\bar{S}^{(N)}\right)^{-1}$. Таким образом, для интеграла (26) получаем выражение

$$
\int_{0}^{\pi} d \zeta\left(C_{m}^{\ell(-)} C_{n}^{\ell(+)}-\bar{S}^{(N)} C_{m}^{\ell(+)} C_{n}^{\ell(+)}-\bar{S}^{(N)^{*}} C_{m}^{\ell(-)} C_{n}^{\ell(-)}+C_{m}^{\ell(+)} C_{n}^{\ell(-)}\right) .
$$

Далее, используя соотношение (16), преобразуем (28) к виду

$$
\begin{aligned}
& \int_{0}^{\pi} d \zeta\left\{C_{m}^{\ell(-)}\left(C_{n}^{\ell(-)}+2 i S_{n}^{\ell}\right)-\bar{S}^{(N)} C_{m}^{\ell(+)} C_{n}^{\ell(+)}-\right. \\
&\left.-\bar{S}^{(N)^{*}} C_{m}^{\ell(-)} C_{n}^{\ell(-)}+C_{m}^{\ell(+)}\left(C_{n}^{\ell(+)}-2 i S_{n}^{\ell}\right)\right\}= \\
&= \int_{0}^{\pi} d \zeta\left\{\left(1-\bar{S}^{(N)^{*}}\right) C_{m}^{\ell(-)} C_{n}^{\ell(-)}+\right. \\
&\left.\quad+\left(1-\bar{S}^{(N)}\right) C_{m}^{\ell(+)} C_{n}^{\ell(+)}+2 i S_{n}^{\ell}\left(C_{m}^{\ell(-)}-C_{m}^{\ell(+)}\right)\right\}= \\
&=\int_{0}^{\pi} d \zeta\left\{\left(1-\bar{S}^{(N)^{*}}\right) C_{m}^{\ell(-)} C_{n}^{\ell(-)}+\left(1-\bar{S}^{(N)}\right) C_{m}^{\ell(+)} C_{n}^{\ell(+)}\right\}+4 \int_{0}^{\pi} d \zeta S_{m}^{\ell} S_{n}^{\ell}
\end{aligned}
$$

Второй интеграл в (29) вычисляем, используя полноту функций $S_{n}^{\ell}[4]$ :

$$
\int_{0}^{\pi} d \zeta S_{n}^{\ell} S_{m}^{\ell}=\delta_{n, m} \frac{\pi}{2} \frac{n !}{(n+2 \ell+1) !(n+\ell+1+\tau)}
$$

где $\tau=\mathcal{Z} b$. Из структуры второго слагаемого в подынтегральном выражении первого интеграла в (29),

$$
\begin{aligned}
\mathcal{F}_{m, n}= & \left(1-\bar{S}^{(N)}\right) C_{m}^{\ell(+)} C_{n}^{\ell(+)}=\frac{p_{N-1}^{\ell}(k) \mathcal{A}_{N}\left(k^{2}\right)-p_{N}^{\ell}(k) \mathcal{B}_{N}\left(k^{2}\right)}{q_{N-1}^{\ell(+)}(k) \mathcal{A}_{N}\left(k^{2}\right)-q_{N}^{\ell(+)}(k) \mathcal{B}_{N}\left(k^{2}\right)} \times \\
& \times\left(\xi-\xi^{-1}\right) q_{m}^{\ell(+)}(k) q_{n}^{\ell(+)}(k)
\end{aligned}
$$

следует

$$
\mathcal{F}_{m, n}(-\zeta)=\left(\mathcal{F}_{m, n}(\zeta)\right)^{*}
$$


т.е. изменение знака (вещественного) $k$ эквивалентно здесь комплексному сопряжению. Действительно, замена $k \rightarrow-k(\zeta \rightarrow-\zeta)$ приводит к замене $\xi \rightarrow \xi^{-1}, t \rightarrow-t$ в (11), т.е. действует так же, как и комплексное сопряжение. Заметим, что вешественные функции $\mathcal{A}_{N}, \mathcal{B}_{N}(18)$ при этом не изменяются. Таким образом, первое слагаемое в подынтегральном выражении первого интеграла в (29) дает вклад

$$
\begin{aligned}
& \int_{0}^{\pi} d \zeta\left(1-\bar{S}^{(N)^{*}}\right) C_{m}^{\ell(-)} C_{n}^{\ell(-)}=\int_{0}^{\pi} d \zeta\left(\mathcal{F}_{m, n}(\zeta)\right)^{*}= \\
& \quad=\int_{0}^{\pi} d \zeta \mathcal{F}_{m, n}(-\zeta)=-\int_{0}^{-\pi} d \zeta \mathcal{F}_{m, n}(\zeta)=\int_{-\pi}^{0} d \zeta \mathcal{F}_{m, n}(\zeta)
\end{aligned}
$$

В результате для интеграла (26) получаем

$$
\begin{aligned}
& \int_{0}^{\pi} d \zeta\left(C_{n}^{\ell(-)}-\bar{S}^{(N)} C_{n}^{\ell(+)}\right)\left(C_{m}^{\ell(-)}-\bar{S}^{(N)} C_{m}^{\ell(+)}\right)^{*}= \\
& \quad=\int_{-\pi}^{\pi} d \zeta \mathcal{F}_{m, n}+\delta_{n, m} 2 \pi \frac{n !}{(n+2 \ell+1) !(n+\ell+1+\tau)}
\end{aligned}
$$

Интеграл в правой части (34) путем замены переменной интегрирования $\zeta \rightarrow \xi=e^{i \zeta}$ (см. (11)) преобразуется в контурный интеграл

$$
-i \oint d \xi \xi^{-1} \mathcal{F}_{m, n}
$$

вдоль единичной окружности в комплексной плоскости $\xi$.

Заметим, что в отсутствие кулоновского взаимодействия детерминант Фредгольма $\mathcal{D}^{(+)}(k)(20)$ является аналитической функцией на всей плоскости $k$, за исключением точки $k=-i b$, где у него имеется полюс порядка $2(N+\ell)[6]$. Следовательно, $S$-матрица имеет полюс в точке $k=i b$ того же порядка. Этот полюс, по-видимому, является следствием асимптотического поведения на бесконечности формфакторов (3) потенциала (5), и его можно отнести к так называемым “ложным” полюсам [11]. Так, например, функция Йоста (детерминант Фредгольма), соответствуюшая экспоненциальному потенциалу $V(r)=V_{0} e^{-r / a}$, имеет полюсы в нижней полуплоскости $k$ в точках $k=-i n / 2 a$, $n=1,2, \ldots$ В свою очередь, $S$-матрица имеет бесконечное множество полюсов в точках $k=i n / 2 a$, которым не соответствуют связанные состояния [7], [11].

Теорема Левинсона устанавливает связь между изменением фазы рассеяния вдоль действительной оси энергии и числом связанных состояний. Фаза рассеяния совпадает с фазой функции Йоста, взятой с обратным знаком [8]. Таким образом, теорема Левинсона может быть сведена к разности между изменением мнимой части $\ln \mathcal{D}^{(+)}(k)$ вдоль замкнутого контура, включаюшего всю действительную ось и полуокружность бесконечного радиуса в верхней полуплоскости, и числом нулей функции Йоста в области, ограниченной этим контуром [7], [8]. Следовательно, полюс $k=i b S$-матрицы, не являющийся нулем детерминанта Фредгольма, а обязанный своим происхождением полюсу $k=-i b$ функции $\mathcal{D}^{(+)}(k)$, не влияет на это соотношение (как и бесконечное число “ложных" полюсов $S$-матрицы, отвечающей экспоненциальному потенциалу). 
Заметим, что преобразование $k \rightarrow \xi$ отображает верхнюю полуплоскость комплексной плоскости $k$ в единичный круг на плоскости $\xi$. Поскольку выражение в знаменателе в правой части (31) с точностью до множителя совпадает с детерминантом Фредгольма $(20)$, то все его корни в верхней полуплоскости $k$ располагаются на мнимой оси [7] (или на вещественной оси плоскости $\xi$ внутри круга единичного радиуса). Из (17), (11) видно, что у подынтегрального выражения в (35) имеется полюс в точке $\xi=0$ порядка $N_{0}=2 N-n-m-1$ (кроме полюсов, отвечающих связанным состояниям). Представляя интеграл в правой части (34) в виде суммы вычетов, тождество (34) можно записать в виде

$$
\begin{aligned}
r_{m, n} \equiv & \left.\operatorname{Res}\right|_{\xi=0} \xi^{-1} \mathcal{F}_{m, n}= \\
= & \frac{b}{\pi} \int_{0}^{\infty} \frac{d k}{b^{2}+k^{2}}\left(C_{m}^{\ell(-)}-\bar{S}^{(N)} C_{m}^{\ell(+)}\right)\left(C_{n}^{\ell(-)}-\bar{S}^{(N)} C_{n}^{\ell(+)}\right)^{*}+ \\
& +\sum_{\nu=1}^{N_{\mathrm{b}}} e^{-i \pi \eta_{\nu}} \exp \left\{\eta_{\nu} \ln \left(\frac{b-\kappa_{\nu}}{b+\kappa_{\nu}}\right)^{2}\right\}\left(\frac{2 b}{b^{2}-\kappa_{\nu}^{2}}\right) \times \\
& \times\left(\frac{b^{2}-\kappa_{\nu}^{2}}{4 b \kappa_{\nu}}\right)^{2 \ell} \frac{\left(\mathcal{M}^{(\nu)}\right)^{2} q_{m}^{\ell(+)}\left(i \kappa_{\nu}\right) q_{n}^{\ell(+)}\left(i \kappa_{\nu}\right)}{\left(\Gamma\left(\ell+1+\eta_{\nu}\right)\right)^{2}}- \\
& -\delta_{n, m} \frac{n !}{(n+2 \ell+1) !(n+\ell+1+\tau)}
\end{aligned}
$$

Обсудим возможность использования тождества (36) в поисках “внешних" параметров. Пусть при некоторых $b$ и $N$ нам удалось описать поведение экспериментальной фазы рассеяния $\delta_{\ell}^{\exp }$ на интервале $\left[0, \epsilon_{0}\right]$. Будем увеличивать $N$ (при фиксированном $b$ ) и следить за разностью

$$
\left|\Im_{m, n}^{(N)}-\Im_{m, n}^{\exp }\right|
$$

где $\Im_{m, n}^{(N)}$ - интеграл в правой части $(36), \Im_{m, n}^{\exp }$ - значение этого интеграла, полученное с использованием $S$-матрицы $\bar{S}=e^{2 i \delta_{\ell}^{\exp }}$. Увеличивая $N$, можно расширять область описания фазы рассеяния (участок, на котором теоретическая фаза $\delta_{\ell}^{(N)}, \bar{S}^{(N)}=e^{2 i \delta_{\ell}^{(N)}}$, совпадает с $\left.\delta^{\exp }\right)$. Таким образом, увеличивая $N$, можно сделать разность (37) сколь угодно малой. Далее, мы не интересуемся поведением фазы рассеяния вне интервала $\left[0, \epsilon_{0}\right]$. Следовательно, можно экстраполировать поведение фазы $\delta_{\ell}^{\exp }(k)$, начиная с $k_{0}=$ $\sqrt{\epsilon_{0}}$, функцией с достаточно быстро затухающей асимптотикой, удовлетворяющей условию [8]

$$
\int^{\infty}\left|\delta_{\ell}(k)\right| \frac{d k}{k}<\infty .
$$

Учитывая возможность произвольного поведения исходной функции $\delta_{\ell}^{\exp }$ вне интервала $\left[0, \epsilon_{0}\right]$, можно ожидать, что переход к модифицированной таким образом экспериментальной фазе улучшит сходимость $\Im_{m, n}^{(N)} \stackrel{N \rightarrow \infty}{\longrightarrow} \Im_{m, n}^{\exp }$.

Заметим, что с ростом $N$ возрастает значение индексов $m, n(m+n \leqslant 2 N-1)$ отличных от нуля вычетов $r_{m, n}(36)$. Можно предположить, что вычеты $r_{m, n}$ с меньшими 
$m, n$ быстрее сходятся к экспериментальным значениям $r_{m, n}^{\exp }$, задаваемым правой частью (36), где вместо $\bar{S}^{(N)}$ используется $e^{2 i \delta_{\ell}^{\exp }}$.

Исходя из вьшесказанного мы предлагаем использовать выражение (36) при достаточно большом $N$ и как можно меньших $m, n$ в качестве уравнения на "внешние" параметры. Для этого $S$-матрица $\bar{S}^{(N)}$ в правой части $(36)$ заменяется модифицированной описанным выше способом экспериментальной $S$-матрицей. Интервал описания исходной экспериментальной фазы остается прежним, однако $N$ должно быть достаточно велико, чтобы воспроизвести модифицированную фазу рассеяния на достаточно широком интервале $\left[0, \epsilon_{1}\right]$ (т.е. теперь неизвестными остаются параметры, "внешние" по отношению к этому новому интервалу).

Заметим, что при задании экспериментальной $S$-матрицы следует учитывать свойства ее образа (17) вблизи точки $k=0$. Так в случае рассеяния нейтральных частиц имеем

$$
1-\bar{S}^{(N)} \sim k^{2 \ell+1}, \quad k \rightarrow 0
$$

В случае заряженных частиц функции (11) в пределе $k=0$ выражаются [5] через вырожденные гипергеометрические функции [12]:

$$
\begin{aligned}
p_{n}^{\ell}(k) & \stackrel{k \rightarrow 0}{\approx}(-1)^{n} \frac{n !}{(n+2 \ell+1) !} L_{n}^{2 \ell+1}(-4 \tau), \\
q_{n}^{\ell(+)}(k) & \stackrel{k \rightarrow 0}{\approx}(-1)^{n} n !(4 \tau)^{2 \ell+1} U(n+2 \ell+2,2 \ell+2,4 \tau) .
\end{aligned}
$$

Таким образом, имеем

$$
1-\bar{S}^{(N)} \sim e^{-2 \pi \mathcal{Z} / k}, \quad k \rightarrow 0
$$

Очевидно, что при соблюдении условий (39) или (41) подынтегральное выражение в правой части (36) не имеет особенностей в начале координат.

\section{5. СВЯЗАННЫЕ КАНАЛЫ}

В "упругом" случае (когда значения энергии в каналах совпадают) многоканальная $S$-матрица, соответствуюшая системе одноименно заряженных частиц с добавочным взаимодействием (1), (2), может быть представлена в виде [13]

$$
\bar{S}^{(\mathcal{N})}=\mathcal{I}-2 i\left[\mathcal{C}_{N-1}^{(+)}-P \mathcal{J} \mathcal{C}_{N}^{(+)}\right]^{-1}\left[\mathcal{S}_{N-1}-P \mathcal{J} \mathcal{S}_{N}\right],
$$

где $\mathcal{I}, \mathcal{C}_{N-1}^{(+)}, \mathcal{C}_{N}^{(+)}, \mathcal{S}_{N-1}, \mathcal{S}_{N}$ и $\mathcal{J}$ - диагональные $\left(N_{\mathrm{c}} \times N_{\mathrm{c}}\right)$-матрищы с элементами:

$$
\begin{gathered}
{[\mathcal{I}]_{\alpha, \beta}=\delta_{\alpha, \beta}, \quad\left[\mathcal{C}_{N-1}^{(+)}\right]_{\alpha, \alpha}=C_{N_{\alpha}-1}^{\ell_{\alpha}(+)}, \quad\left[\mathcal{C}_{N}^{(+)}\right]_{\alpha, \alpha}=C_{N_{\alpha}}^{\ell_{\alpha}(+)}} \\
{\left[\mathcal{S}_{N-1}\right]_{\alpha, \alpha}=S_{N_{\alpha}-1}^{\ell_{\alpha}}, \quad\left[\mathcal{S}_{N}\right]_{\alpha, \alpha}=S_{N_{\alpha}}^{\ell_{\alpha}}} \\
{[\mathcal{J}]_{\alpha, \alpha}=\frac{\left(N_{\alpha}+2 \ell_{\alpha}+1\right) !}{2 b\left(N_{\alpha}-1\right) !}\left(b^{2}+k^{2}\right)}
\end{gathered}
$$


Элементы $P$-матрицы имеют вид [2]

$$
P_{\alpha, \beta}=d_{\alpha} d_{\beta} \sum_{j=1}^{\mathcal{N}} \frac{Z_{K_{\alpha}, j} Z_{K_{\beta}, j}}{k^{2}-\lambda_{j}}, \quad d_{\alpha}=d_{N_{\alpha}-1}^{\ell_{\alpha}}
$$

Для описания связанных состояний воспользуемся выражением для детерминанта Фредгольма

$$
\mathcal{D}^{(+)}(k)=\operatorname{det}\left[\mathcal{Q}_{N-1}^{(+)}-P \mathcal{J} \mathcal{Q}_{N}^{(+)}\right] \prod_{j=1}^{\mathcal{N}} \frac{k^{2}-\lambda_{j}}{k^{2}+b^{2}} \prod_{\alpha=1}^{N_{c}} \frac{-\left(N_{\alpha}+2 \ell_{\alpha}+1\right) !}{\left(N_{\alpha}-1\right) !\left(2 \ell_{\alpha}+1\right) !}
$$

где $\mathcal{Q}_{N-1}^{(+)}, \mathcal{Q}_{N}^{(+)}-$диагональные матрицы:

$$
\left[\mathcal{Q}_{N-1}^{(+)}\right]_{\alpha, \alpha}=q_{N_{\alpha}-1}^{\ell_{\alpha}(+)}(k), \quad\left[\mathcal{Q}_{N}^{(+)}\right]_{\alpha, \alpha}=q_{N_{\alpha}}^{\ell_{\alpha}(+)}(k) .
$$

Тогда условие (7) преобразуется в уравнение

$$
\left.\operatorname{det}\left[\mathcal{Q}_{N-1}^{(+)}-P \mathcal{J} \mathcal{Q}_{N}^{(+)}\right]\right|_{k=i \kappa}=0 .
$$

Выражение для вычетов элементов многоканальной $S$-матрицы $S=e^{i \sigma_{L}} \bar{S}^{(\mathcal{N})} e^{i \sigma_{L}}$ имеет вид [9]

$$
\left.i \operatorname{Res}\right|_{k=i \kappa}[S(k)]_{\alpha, \beta}=e^{i \pi\left(\ell_{\alpha}+\ell_{\beta}\right) / 2} \mathcal{M}_{\alpha} \mathcal{M}_{\beta},
$$

где $e^{i \sigma_{L}}-$ диагональная матрица с элементами

$$
\left[e^{i \sigma_{L}}\right]_{\alpha, \alpha}=\frac{\Gamma\left(\ell_{\alpha}+1+i t\right)}{\left|\Gamma\left(\ell_{\alpha}+1+i t\right)\right|}
$$

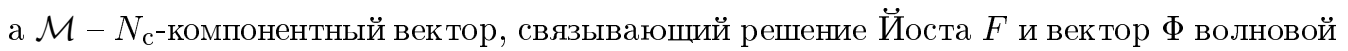
функции связанного состояния [7]:

$$
F(i \kappa, r) \mathcal{M}=\Phi(i \kappa, r)
$$

Из (42), (48) следует уравнение

$$
\begin{aligned}
& \left.i \operatorname{Res}\right|_{k=i \kappa}\left[\left[\mathcal{Q}_{N-1}^{(+)}-P \mathcal{J} \mathcal{Q}_{N}^{(+)}\right]^{-1}\left[\mathcal{P}_{N-1}-P \mathcal{J} \mathcal{P}_{N}\right]\right]_{\alpha, \beta}= \\
& \quad=e^{-i \pi \eta} \exp \left\{\eta \ln \left(\frac{b-\kappa}{b+\kappa}\right)^{2}\right\}\left(\frac{b^{2}-\kappa^{2}}{4 b \kappa}\right)^{\ell_{\alpha}+\ell_{\beta}+1} \frac{\mathcal{M}_{\alpha}}{\Gamma\left(\ell_{\alpha}+1+\eta\right)} \frac{\mathcal{M}_{\beta}}{\Gamma\left(\ell_{\beta}+1+\eta\right)}
\end{aligned}
$$

Дополнительные уравнения могут быть получены путем учета полюсной особенности элементов $S$-матрицы (42) в точке $k=i b$. Для этого потребуются матричные аналоги интегралов (26)

$$
\begin{gathered}
\int_{0}^{\pi} d \zeta\left[\mathcal{C}_{m}^{(-)}-\mathcal{C}_{m}^{(+)} \bar{S}^{(\mathcal{N})}\right]\left[\mathcal{C}_{n}^{(-)}-\mathcal{C}_{n}^{(+)} \bar{S}^{(\mathcal{N})}\right]^{\dagger}= \\
=\int_{-\pi}^{\pi} d \zeta \mathcal{C}_{m}^{(+)}\left[\mathcal{I}-\bar{S}^{(\mathcal{N})}\right] \mathcal{C}_{n}^{(+)}+2 \pi \Delta_{m, n}
\end{gathered}
$$


где элементы диагональных матриц $\mathcal{C}_{n}^{(+)}, \Delta_{m, n}$ задаются выражениями

$$
\begin{aligned}
{\left[\mathcal{C}_{n}^{(+)}\right]_{\alpha, \alpha} } & =C_{n_{\alpha}}^{\ell_{\alpha}(+)} \\
{\left[\Delta_{m, n}\right]_{\alpha, \alpha} } & =\delta_{m_{\alpha}, n_{\alpha}} \frac{n_{\alpha} !}{\left(n_{\alpha}+2 \ell_{\alpha}+1\right) !\left(n_{\alpha}+\ell_{\alpha}+1+\tau\right)} .
\end{aligned}
$$

При выводе (52) мы воспользовались соотношением

$$
\mathcal{C}_{n}^{(+)}=\Omega^{-1} \mathcal{Q}_{n}^{(+)}, \quad \mathcal{S}_{n}=\sin \zeta \Omega \mathcal{P}_{n}
$$

где $\mathcal{Q}_{n}^{(+)}, \mathcal{P}_{n}^{(+)}$и $\Omega$ - диагональные матрицы с элементами

$$
\begin{aligned}
{\left[\mathcal{Q}_{n}^{(+)}\right]_{\alpha, \alpha} } & =q_{n_{\alpha}}^{\ell_{\alpha}(+)}(k), \quad\left[\mathcal{P}_{n}\right]_{\alpha, \alpha}=p_{n_{\alpha}}^{\ell_{\alpha}}(k), \\
{[\Omega]_{\alpha, \alpha} } & =\frac{(2 \sin \zeta)^{\ell_{\alpha}}\left|\Gamma\left(\ell_{\alpha}+1+i t\right)\right|}{e^{\pi t / 2} \xi^{i t}} .
\end{aligned}
$$

Подынтегральное выражение в правой части (52) преобразуется к виду

$$
\mathcal{F}_{m, n}=\left(\xi-\xi^{-1}\right) \mathcal{Q}_{m}^{(+)}\left[\mathcal{Q}_{N-1}^{(+)}-P \mathcal{J} \mathcal{Q}_{N}^{(+)}\right]^{-1}\left[\mathcal{P}_{N-1}-P \mathcal{J} \mathcal{P}_{N}\right] \mathcal{Q}_{n}^{(+)}
$$

А сам интеграл представляется в виде контурного (35) и сводится к сумме вычетов по связанным состояниям и вычету, соответствуюшему полюсу порядка $N_{0}=N_{\alpha}+N_{\beta}-$ $m_{\alpha}-n_{\beta}-1$ в точке $\xi=0$. В результате получаем тождество, аналогичное $(36)$ :

$$
\begin{aligned}
\operatorname{Res} & \left.\right|_{\xi=0} \xi^{-1}\left[\mathcal{F}_{m, n}\right]_{\alpha, \beta}=\frac{b}{\pi} \int_{0}^{\infty} \frac{d k}{b^{2}+k^{2}}\left[\left[\mathcal{C}_{m}^{(-)}-\mathcal{C}_{m}^{(+)} \bar{S}^{(\mathcal{N})}\right]\left[\mathcal{C}_{n}^{(-)}-\mathcal{C}_{n}^{(+)} \bar{S}^{(\mathcal{N})}\right]^{\dagger}\right]_{\alpha, \beta}- \\
& -\left[\Delta_{m, n}\right]_{\alpha, \beta}+\sum_{\nu=1}^{N_{\mathrm{b}}} e^{-i \pi \eta_{\nu}} \exp \left\{\eta_{\nu} \ln \left(\frac{b-\kappa_{\nu}}{b+\kappa_{\nu}}\right)^{2}\right\}\left(\frac{2 b}{b^{2}-\kappa_{\nu}^{2}}\right) \times \\
& \times\left(\frac{b^{2}-\kappa_{\nu}^{2}}{4 b \kappa_{\nu}}\right)^{\ell_{\alpha}+\ell_{\beta}} \frac{\mathcal{M}_{\alpha}^{(\nu)} q_{m_{\alpha}}^{\ell_{\alpha}(+)}\left(i \kappa_{\nu}\right)}{\Gamma\left(\ell_{\alpha}+1+\eta_{\nu}\right)} \frac{\mathcal{M}_{\beta}^{(\nu)} q_{n_{\beta}}^{\ell_{\beta}(+)}\left(i \kappa_{\nu}\right)}{\Gamma\left(\ell_{\beta}+1+\eta_{\nu}\right)} .
\end{aligned}
$$

\section{6. ПРИМЕР}

Процедуру восстановления потенциала (5) проиллюстрируем на примере, где в качестве входной информации используются данные по $s$-волновому рассеянию нейтральной частицы на сферической прямоугольной потенциальной яме глубиной $V_{0}$ и радиусом $R$. Параметр ямы $K_{0} R=2, K_{0}=\sqrt{2 m V_{0}} / \hbar$, задает связанное состояние с энергией $E=-\kappa^{2}, \kappa R=0.638045$ и асимптотической нормировочной константой $\mathcal{M} R^{1 / 2}=$ 1.583324. Мы ограничились максимальным значением $k_{0} R=6$, т.е. $\epsilon_{0} R^{2}=36$.

"Внутренние" параметры $\left\{\lambda_{j} \in\left[0, \epsilon_{0}\right]\right\}$ совпадают с полюсами вспомогательной функции $\widetilde{P}_{N}(\epsilon)$, которую получаем, обращая выражение (13) относительно функции $P_{N}\left(k^{2}\right)$ (15):

$$
\widetilde{P}_{N}(\epsilon)=\frac{1}{J_{N-1, N}(k)} \frac{C_{N-1}^{0(-)}-C_{N-1}^{0(+)} \bar{S}}{C_{N}^{0(-)}-C_{N}^{0(+)} \bar{S}}
$$


В свою очередь, вычеты $\widetilde{P}_{N}$ отождествляются с $\left\{\left(d_{N-1}^{0} Z_{N, j}\right)^{2}\right\}$ [2]. Например, для $N=$ 4 и $b R=4.65$ получаем следующие две пары "внутренних" параметров:

$$
\begin{array}{ll}
\lambda_{1} R^{2}=9.080989, & Z_{4,1}^{2}=0.173225 \\
\lambda_{2} R^{2}=35.787885, & Z_{4,2}^{2}=0.355379 .
\end{array}
$$

Таким образом, данным $N$ и $b$ отвечает минимальное (с учетом необходимости описания связанного состояния) число "внешних" параметров: $\left\{\lambda_{3}, Z_{4,3}, \lambda_{4}, Z_{4,4}\right\}$. Для их вычисления строим систему, состояшую из следуюших условий: 1) условия нормировки (6); $2)$ условия $(21) ; 3)$ уравнения $(25) ; 4)$ уравнения $(36)$, которое в данном примере, где

$$
C_{n}^{0( \pm)}=-\frac{1}{n+1}\left(\frac{k \mp i b}{k \pm i b}\right)^{n+1}
$$

принимает вид

$$
\begin{gathered}
-\left.i \frac{1}{\mathcal{K} !} \frac{d^{\mathcal{K}}}{d k^{\mathcal{K}}}\left\{\frac{(N+1) \mathcal{A}_{N}\left(k^{2}\right)-N((k+i b) /(k-i b)) \mathcal{B}_{N}\left(k^{2}\right)}{(N+1) \mathcal{A}_{N}\left(k^{2}\right)-N((k-i b) /(k+i b)) \mathcal{B}_{N}\left(k^{2}\right)}(k+i b)^{\mathcal{K}-1}\right\}\right|_{k=i b}= \\
=\frac{1}{2 \pi} \int_{-\infty}^{\infty} \frac{d k}{b^{2}+k^{2}}(1-\bar{S})\left(\frac{k-i b}{k+i b}\right)^{m+n+2}+\frac{1}{b^{2}-\kappa^{2}}\left(\frac{\kappa-b}{\kappa+b}\right)^{m+n+2} \mathcal{M}^{2} \\
\mathcal{K}=2 N-m-n-2 .
\end{gathered}
$$

В правой части используется $S$-матрица $\bar{S}=e^{2 i \bar{\delta}}$, где фаза $\bar{\delta}(k)$ совпадает с экспериментальной фазой $\delta^{\exp }(k)$ на интервале $\left[0, k_{0}\right]$, а в области $k>k_{0}$ экстраполирована рациональной функцией с затухающей $\sim 1 / k[8]$ асимптотикой:

$$
\bar{\delta}(k)=\left\{\begin{array}{cl}
\delta^{\exp }(k), & k \leqslant k_{0}, \\
\frac{u}{k^{2}}+\frac{v}{k}, & k>k_{0} .
\end{array}\right.
$$

Поведение исходной экспериментальной $\delta^{\exp }(k)$ и модифицированной $\bar{\delta}(k)$ фаз рассеяния (при $u=9.717254, v=0.315288$ ) внутри интервала $\left[0, \epsilon_{0}\right]$ и за его пределами показано на рисунке. Здесь сплошная линия соответствует модифицированной фазе, а сплошная тонкая линия - экспериментальной.

Результат решения системы (1)-(4) с $m=0$ и $n=2$ таков:

$$
\begin{array}{ll}
\lambda_{3} R^{2}=203.292303, & Z_{4,3}^{2}=0.454214 \\
\lambda_{4} R^{2}=-0,0442675, & Z_{4,4}^{2}=0.0171819 .
\end{array}
$$

Далее по собственным значениям $\left\{\lambda_{j}\right\}$ и квадратам элементов $\left\{Z_{N, j}\right\}$ последней строки матрицы $\mathbf{Z}$ собственных векторов строится (например, трехдиагональная) матрица гамильтониана [1]. Вычитанием из последней матрицы оператора кинетической энергии 

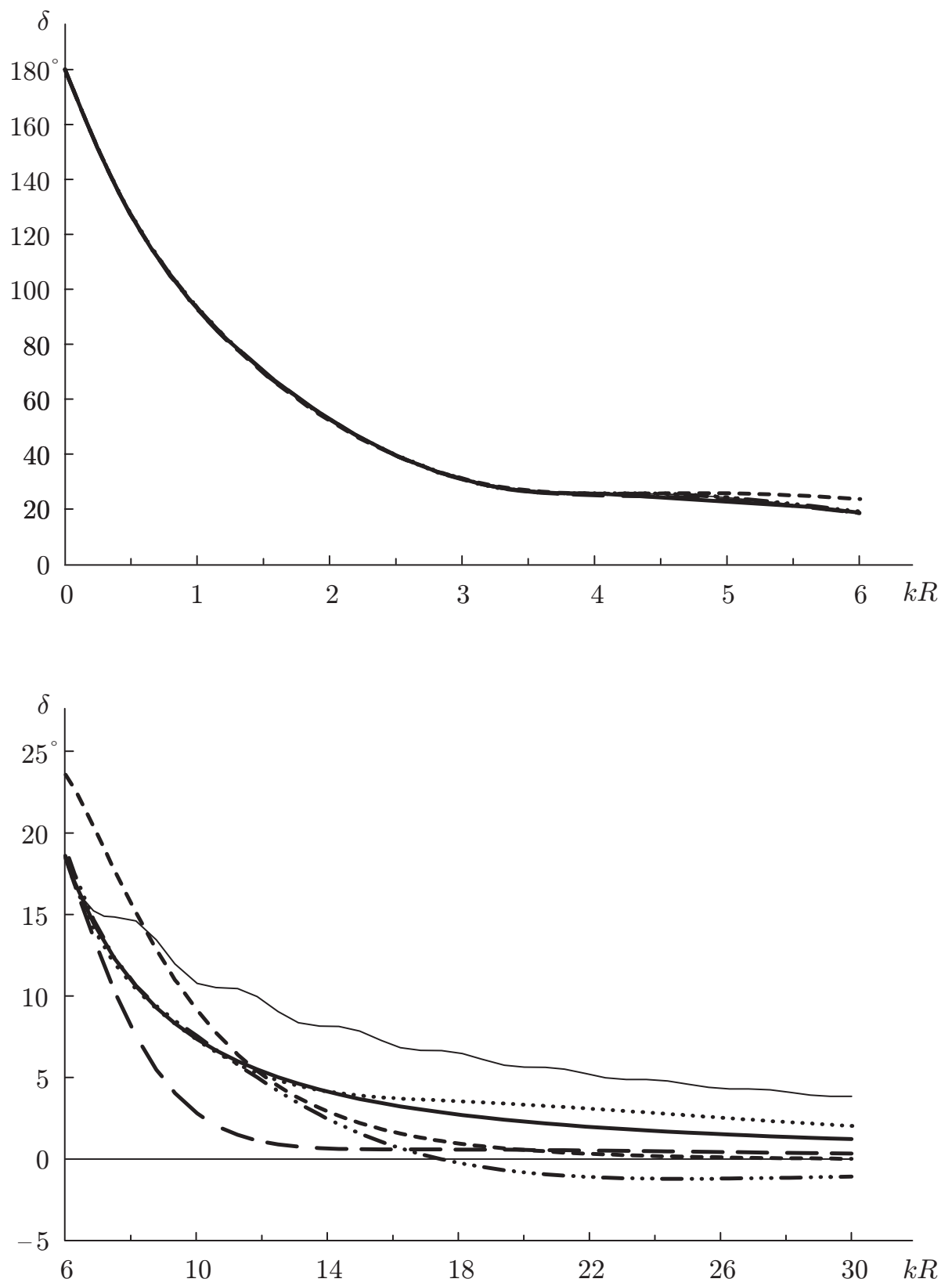

получают матрицу $\mathbf{V}$ потенциала (5) [2]. Теоретическая фаза рассеяния $\delta^{(4)}(k)$ представлена на рисунке штриховой линией с длинными штрихами.

Эволюцию теоретической фазы рассеяния $\delta^{(N)}$ при возрастании $N$ от $N=3$ до $N=6$ можно проследить на рисунке, где фазы $\delta^{(3)}(k), \delta^{(5)}(k)$ и $\delta^{(6)}(k)$ изображены штриховой с короткими штрихами, штрихпунктирной и пунктирной линиями, соответственно. Здесь при всех $N$ число “внешних" параметров остается фиксированным: $\left\{\lambda_{N-1}\right.$, 
$\left.Z_{N, N-1}\right\}$ и $\left\{\lambda_{N}, Z_{N, N}\right\}$. Неизвестные параметры были найдены в результате решения системы уравнений (1)-(4); при этом в уравнении (61) использовалась своя для каждого $N$ комбинация индексов $m=0$ и $n=n_{0}$, указанная в таблице. Таблица содержит значения вычетов $r_{m=0, n}(36)$, соответствуюших теоретическим $S$-матрицам $\bar{S}^{(N)}(13)$. Для сравнения в таблице также приводятся значения $r_{m, n}^{\exp }$, задаваемые правой частью $(36)$ с $S$-матрицей $\bar{S}=e^{2 i \bar{\delta}}$, где модифицированная фаза $\bar{\delta}$ определена выражением (62). Видно, что с ростом $N$ (по мере того как увеличивается интервал, где теоретическая фаза совпадает с модифицированной экспериментальной) значения вычетов $r_{m, n}$ приближаются к экспериментальным значениям $r_{m, n}^{\exp }$.

\begin{tabular}{|c|c|c|c|c|c|}
\hline$n$ & $r_{0, n}^{\text {exp }}$ & \multicolumn{4}{|c|}{$r_{0, n}$} \\
\cline { 3 - 6 } & & $N=3$, & $N=4$, & $N=5$, & $N=6$, \\
& & $n_{0}=1$ & $n_{0}=2$ & $n_{0}=5$ & $n_{0}=7$ \\
\hline 0 & 11.402843 & 11.254276 & 11.588794 & 11.469717 & 11.384319 \\
1 & -1.077483 & -1.077483 & -1.013361 & -1.047574 & -1.076793 \\
2 & 0.326500 & 0.3956003 & 0.326500 & 0.360071 & 0.314934 \\
3 & -0.0288681 & -0.00743774 & -0.0405596 & -0.0153991 & -0.0335707 \\
4 & -0.0462000 & -0.0483459 & -0.0604831 & -0.0407937 & -0.0494786 \\
5 & 0.0164725 & 0 & 0.00789452 & 0.0164725 & 0.0161637 \\
6 & 0.0104322 & 0 & 0.0121261 & 0.00781545 & 0.0114440 \\
7 & -0.00458193 & 0 & 0 & -0.00626561 & -0.00458193 \\
8 & -0.00323731 & 0 & 0 & -0.00744598 & -0.00191864 \\
9 & 0.00137365 & 0 & 0 & 0 & 0.00209275 \\
10 & 0.00144027 & 0 & 0 & 0 & 0.00363561 \\
\hline
\end{tabular}

\section{7. ЗАКЛЮЧЕНИЕ}

В работе [2] был предложен приближенный метод решения обратной задачи рассеяния одноименно заряженных частиц с короткодействуюшим добавочным потенциалом, представленным сепарабельным разложением с использованием лагерровских формфакторов. Искомый потенциал конечного ранга описывает данные рассеяния на ограниченном интервале энергий. В процедуре восстановления потенциала [2] используется информация о рассеянии (кроме связанных состояний) лишь из данного энергетического интервала. Следствием этого ограничения является присутствие свободных параметров. В задаче потенциального рассеяния выбором значений ранга $N$ потенциала (5) и масштабного параметра $b$ число свободных параметров можно сократить до единицы. В многоканальной задаче количество свободных параметров возрастает с ростом числа каналов по квадратичному закону, и их определение представляет проблему. В 
данной работе предлагается способ учета данных рассеяния, основанный на свойствах $S$-матришы, который позволяет устранить эту неопределенность.

Благодарности. Автор выражает признательность А. М. Широкову за стимулирующие дискуссии.

\section{Список литературы}

[1] C. A. Зайщев. ТМФ. 1998. Т. 115. С. 263; 1999. Т. 121. С. 424.

[2] S. A. Zaitsev, E. I. Kramar. J. Phys. G. 2001. V. 27. P. 2037.

[3] H. A. Yamani, L. Fishman. J. Math. Phys. 1975. V. 16. P. 410.

[4] H. A. Yamani, M. S. Abdelmonem. J. Phys. B. 1997. V. 30. P. 1633.

[5] J. T. Broad. Phys. Rev. A. 1985. V. 31. P. 1494.

[6] S. A. Zaitsev, E. I. Kramar. J-matrix method and Bargmann potentials. quant-ph/0107109.

[7] Р. Ньютон. Теория рассеяния волн и частиц. М.: Мир, 1969.

[8] К. Шадан, П. Сабатье. Обратные задачи в квантовой теории рассеяния. М.: Мир, 1980.

[9] Л. Д. Блохинцев, И. Борбей, Э. И. Долинский. ЭЧАЯ. 1977. Т. 8. № 6. С. 1189.

[10] Б. Н. Захарьев, А. А. Сузько. Потенциалы и квантовое рассеяние. Прямая и обратная задачи. М.: Энергоатомиздат, 1985.

[11] А. И. Базь, Я. Б. Зельдович, А. М. Переломов. Рассеяние, реакции и распады в нерелятивистской квантовой механике. М.: Наука, 1971.

[12] Справочник по специальным функциям. Под редакцией М. Абрамовица и И. Стиган. М.: Наука, 1979.

[13] J. M. Bang, A.I. Mazur, A. M. Shirokov, Yu.F. Smirnov, S. A. Zaytsev. Ann. Phys. 2000. V. 280. P. 299. 